\title{
Correction to: Virtual Power Plant Model
}

\section{Correction to:}

Chapter 2 in: L. Baringo, M. Rahimiyan, Virtual Power Plants and Electricity Markets, https://doi.org/10.1007/978-3-030-47602-1_2

The original version of the chapter was inadvertently published with wrong placement of section headings and an incomplete GAMS code. The chapter has now been corrected and approved by the author. 